\title{
Modeling brain connectivity dynamics in functional magnetic resonance imaging via particle filtering
}

\author{
Pierfrancesco Ambrosi ${ }^{1 *}$ (D, Mauro Costagli2,3, Ercan E. Kuruoğlu, ${ }^{4,6}$, Laura Biagi ${ }^{3,5}$, Guido Buonincontri3,5 and \\ Michela Tosetti ${ }^{3,5}$
}

\begin{abstract}
Interest in the studying of functional connections in the brain has grown considerably in the last decades, as many studies have pointed out that alterations in the interaction among brain areas can play a role as markers of neurological diseases. Most studies in this field treat the brain network as a system of connections stationary in time, but dynamic features of brain connectivity can provide useful information, both on physiology and pathological conditions of the brain. In this paper, we propose the application of a computational methodology, named Particle Filter (PF), to study non-stationarities in brain connectivity in functional Magnetic Resonance Imaging (fMRI). The PF algorithm estimates time-varying hidden parameters of a first-order linear time-varying Vector Autoregressive model (VAR) through a Sequential Monte Carlo strategy. On simulated time series, the PF approach effectively detected and enabled to follow time-varying hidden parameters and it captured causal relationships among signals. The method was also applied to real fMRI data, acquired in presence of periodic tactile or visual stimulations, in different sessions. On these data, the PF estimates were consistent with current knowledge on brain functioning. Most importantly, the approach enabled to detect statistically significant modulations in the cause-effect relationship between brain areas, which correlated with the underlying visual stimulation pattern presented during the acquisition.
\end{abstract}

Keywords: Brain connectivity, fMRI, Particle filter, Sequential Monte Carlo, VAR model

\section{Introduction}

The understanding of brain functioning is linked to the study of the dynamic interaction among anatomically segregated brain areas. These interactions are labeled functional and effective connectivity and refer to distinct ways of considering connections among brain region. While complementary to structural connectivity, which describes anatomical connections between brain regions [1], they concern functional connections that are not necessarily achieved through a direct anatomical link between brain areas. Functional connectivity regards

\footnotetext{
*Correspondence: pfa2804@gmail.com

${ }^{1}$ Department of Neuroscience, Psychology, Pharmacology and Child

Health, University of Florence, Florence, Italy

Full list of author information is available at the end of the article
}

connections as statistical codependencies between the signal of different brain regions, and consequently it is a non-directional and model-free description of the brain network. On the contrary, effective connectivity defines the temporal relationship and causal influences among brain regions in a given network model [2].

Functional Magnetic Resonance Imaging (fMRI) is frequently employed in brain connectivity studies, given its non-invasiveness and satisfactory spatiotemporal resolution, both in physiology and pathology (e.g. Alzheimer's disease [3-5], schizophrenia [6] and Major Depression Disorder [7]). From brain connectivity studies it emerged that brain dynamics, in particular effective connectivity, may provide a biological marker for specific brain disease and a tool for monitoring responses to treatments of these pathologies [8-11].

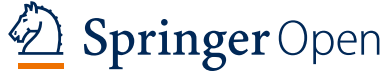

(c) The Author(s) 2021. Open Access This article is licensed under a Creative Commons Attribution 4.0 International License, which permits use, sharing, adaptation, distribution and reproduction in any medium or format, as long as you give appropriate credit to the original author(s) and the source, provide a link to the Creative Commons licence, and indicate if changes were made. The images or other third party material in this article are included in the article's Creative Commons licence, unless indicated otherwise in a credit line to the material. If material is not included in the article's Creative Commons licence and your intended use is not permitted by statutory regulation or exceeds the permitted use, you will need to obtain permission directly from the copyright holder. To view a copy of this licence, visit http://creativecommons.org/licenses/by/4.0/. 
Granger Causality (GC) and Dynamic Causal Modeling (DCM) are methods to investigate effective connectivity. Granger causality is present when knowledge on temporal evolution of the signal in a certain brain region $A$ improves the predictability of another brain region $B[12$, 13]. This approach is based on the evaluation of a linear codependence among time series, and it is therefore limited to a stationary framework or needs a sliding-window approach to address time-varying coupling between regions, which has limitations [14]. Differently, in DCM the predicted relationship between neural activity and observed fMRI signal needs to be specified in a predetermined model, hence requiring previous knowledge about the timing and effect on signals of the connectivity modulation [15].

The Sequential Monte Carlo (SMC) methodology [16] is crucially different from these two strategies. SMC approaches estimate the hidden states of a dynamic system with only partial and noisy observations, without further assumptions on the presence of variations in connectivity. A specific SMC methodology called Particle Filter (PF) employs Monte Carlo sampling to approximate probability density functions and it updates the posteriors with the arrival of new samples.

The SMC algorithm proposed here was recently developed by Ancherbak et al. [17], originally for time-varying gene network modeling. We adapted it for the study of brain connectivity using fMRI data and the feasibility and behaviour of the proposed approach has been studied on synthetic data mimicking fMRI time-series. When applied to real fMRI datasets, results were compared to correlation between delayed time series, considered as a proxy measure for stationary effective connectivity. Two different experimental paradigms were tested: the first one, whose preliminary results were presented in abstract form [18], involved tactile stimulation during an fMRI acquisition with temporal resolution of $2 \mathrm{~s}$. The second experiment employed a periodic visual stimulation with significantly improved time resolution of $0.8 \mathrm{~s}$.

\section{Methodology}

\subsection{Model and algorithm}

Particle filter [17, 19-22] is a sequential Monte Carlo methodology based on the Bayes theorem on conditional probability. Particle filters estimate the probability distributions of hidden variables of interest, modeled according to a hypothesized state-space equation. The probability density function (pdf) of the hidden variables is allowed to be time-varying and is therefore sequentially updated when new data become available. Such probability distribution is estimated from the data, modeled according to a hypothesized observation equation. In brain connectivity studies based on fMRI data, the relationship among the time-series of $R$ different brain Regions of Interest (ROIs) $\mathbf{x}_{t}=\left\{x_{1}(t), \ldots, x_{R}(t)\right\}$ can be modeled as a first order linear Vector Autoregressive (VAR) model [12, 21, 23-25] as:

$$
x_{i}(t)=\sum_{j}^{R} a_{i j}(t) x_{j}(t-1)+\eta_{i}(t) \quad i=1, \ldots, R
$$

or in matrix notation:

$$
\mathbf{x}(t)=\mathbf{a}(t) \mathbf{x}(t-1)+\boldsymbol{\eta}(t)
$$

where

$$
\mathbf{a}(t)=\left[\begin{array}{cccc}
a_{11}(t) & a_{12}(t) & \ldots & a_{1 R}(t) \\
a_{21}(t) & a_{22}(t) & \ldots & a_{2 R}(t) \\
\vdots & \vdots & \ddots & \vdots \\
a_{R 1}(t) & a_{R 2}(t) & \ldots & a_{R R}(t)
\end{array}\right]
$$

which is employed as the observation equation describing the relationship between the observations $\mathbf{x}(t)$ at time $t$ and those at time $t-1$ (that is, $\mathbf{x}(t-1)$ ); $\boldsymbol{\eta}(t)$ is the vector of observation noise; the matrix of hidden parameters of interest $\mathbf{a}(t)$ represents the causal influence exerted between different areas, and its elements $a_{i} j(t)$ are the coefficients which represent conditional dependence. In particular, it can be assumed that elements of $\mathbf{a}(t)$ are allowed to be time-varying:

$$
a_{i j}(t)=a_{i j}(t-1)+v_{i j}(t)
$$

where $a_{i j}(t)$ is the $i j$ th element of the matrix $\mathbf{a}(t)$, describing the influence of the $j$ th region over the $i$ th region, and $v_{i j}(t)$ is the process noise (innovation) term.

The adoption of a linear model was supported by the well-established body of literature on fMRI data modelling and brain connectivity analysis at the temporal scales of fMRI data $[25,26]$. The adopted autoregressive model was first-order, which was optimal on the basis of the Schwartz criterion, in accordance with previous findings $[12,25,27]$.

The PF algorithm evolves from an initial probability distribution for $a_{i j}(t-1)$, which we chose to be uniform at $t=1$, and through Eq. (4) it generates new possible values for $a_{i j}(t)$. The $\mathrm{N}$ particles are generated from the probability distributions of the elements of $\mathbf{a}_{i}(t)$ : the distributions are adapted with each new observation through the mechanism provided by particle filtering, to describe for the set of coefficients $a_{i j}$ at every timestep. The algorithm generates $\mathrm{N}$ particles, by updating those at the previous time point (initialized to zero at $\mathrm{t}=0$ ) using a noise innovation term, by following Eq. 4 . In our implementation, the innovation values are drawn from a Gaussian distribution. The standard deviation of this distribution follows the absolute difference between 
the two previous coefficient values estimated by the algorithm and constrained between a minimum of 0.1 and a maximum of 0.4 : these values were chosen empirically to prevent divergence and, at the same time, capture non-stationarities.

With Eq. (2), the PF algorithm generates predicted values of the observations at time $t$. The desired probability density function of the parameters of interest $\mathbf{a}(t)$ can be estimated via Bayes theorem as follows:

$$
p(\mathbf{a}(t) \mid \mathbf{x}(1, \ldots, t))=\frac{p(\mathbf{x}(t) \mid \mathbf{a}(t)) p(\mathbf{a}(t) \mid \mathbf{x}(1, \ldots, t-1))}{p(\mathbf{x}(t) \mid \mathbf{x}(1, \ldots, t-1))}
$$

and with the assumption of Gaussian noise we have

$$
p\left(x_{i}(t) \mid \mathbf{a}_{i}(t)\right)=\frac{1}{\left(2 \pi \sigma_{\eta}^{2}\right)^{R / 2}} \exp \left(-\frac{\left(x_{i}(t)-\hat{x}_{i}(t)\right)^{2}}{2 \sigma_{\eta}^{2}}\right)
$$

where $\hat{x}_{i}(t)$ are the data estimated through Eq. (1) at time $t$ for the $i$-th ROI and $\mathbf{a}_{i}(t)=\left\{a_{i 1}, \ldots, a_{i R}\right\}$ is the vector of hidden variables associated with the $i$-th ROI at time $t$, that is, the $i$-th row of matrix $\mathbf{a}_{t}$. In Eq. 6 the value of the $\mathbf{a}_{i}(t)$ elements determines the value of the estimate $\hat{x}_{i}(t)$, and the weight of the $i$-th particle.

In most applications, Eq. (5) cannot be solved analytically [28], but it can be computed through the Sequential Monte Carlo sampling scheme, which consists in representing the pdf $p(\mathbf{a}(t) \mid \mathbf{x}(1, \ldots, t))$ as a discrete set of $\mathrm{N}$ particles:

$$
p\left(\mathbf{a}_{i}(t) \mid \mathbf{x}(1, \ldots, t)\right) \approx \sum_{n=1}^{N} w_{t}^{(n)} \delta\left(\mathbf{a}_{i}(t)-\mathbf{a}_{i}^{(n)}(t)\right)
$$

where $w_{t}^{(n)}$ is the weight associated to the $n$-th particle vector $\mathbf{a}_{i}^{(n)}(t)$ for the $i$ th row of matrix $\mathbf{a}(t)$ at time $t$. The Sequential Importance Sampling (SIS) [28] methodology provides a strategy to compute the weights. It has been shown [20] that the weights can be sequentially updated as follows:

$$
w_{t}^{(n)} \propto w_{t-1}^{(n)} p\left(\mathbf{x}(t) \mid \mathbf{a}_{i}(t)^{(n)}\right)
$$

where the proportionality takes into account normalization factors. With this approach, at each time instant $t$ we have a sample set $\left\{\mathbf{a}_{i}(t)^{(n)}, w_{t}^{(n)}\right\}$ for $n=1, \ldots, N$ and for $i=1, \ldots, R$ which can be used to estimate the pdf of the parameters and to infer information about the network. However, after some iterations, most of the particles will have a very low statistical weight, resulting in a lower exploration efficiency of the algorithm. To overcome this typical problem of sequential Monte Carlo methodologies, a step called Resampling is performed. The number of effective particles was defined in [29] as

$$
N_{\text {eff }}=\frac{1}{\sum_{n=1}^{N}\left(w_{t}^{(n)}\right)^{2}}
$$

If $N_{\text {eff }}$ is below a certain arbitrary threshold the Resampling is performed: particles with weight below a certain threshold are substituted by copies of particles with sufficiently high weights and to each of the new particles set is assigned the same weight $1 / N$. This results in a more effective exploration of the solution space, because only statistically relevant particles remain after this step. The VAR process estimation problem using SMC was developed in [30], and it was extended and applied to timevarying gene expression networks in [17].

To sum up, the resulting algorithm can be schematically expressed as in Table 1 . In our implementation the procedure is repeated $N_{r}=100$ times, all independent from each other, to provide a better exploration of the solution space, and resampling was performed when

\begin{tabular}{|c|c|}
\hline Input the BOLD fMRI data series & $\mathbf{x}_{t}$ \\
\hline Set the number of particles & $N(=2000$ in our case $)$ \\
\hline Set starting point for coefficients values & $\mathbf{a}_{t=0}=0$ \\
\hline Start PF & fort $=1: T$ \\
\hline updating step & $\begin{array}{l}\text { generate } N \text { particles from previous coefficients' values through } \\
\qquad a_{i j}(t)=a_{i j}(t-1)+v_{i j}(t)\end{array}$ \\
\hline estimation step & $\begin{array}{l}\text { predict the values of the observations at time } t \text { from } \\
\text { values at time } t-1 \text { with } \mathbf{x}(t)=\mathbf{a}(t) \mathbf{x}(t-1)+\boldsymbol{\eta}(t) \\
\text { compute the likelihood between predicted values and } \\
\text { observed values with (6) normalize the weights and resample }\end{array}$ \\
\hline End PF & end for on $t$ \\
\hline
\end{tabular}
$N_{\text {eff }}<30 \%$ of the total number of particles. The final outputs of the algorithm are the $\mathbf{a}_{t}$ computed as the average of the $N_{r}$ repetitions. In this implementation, the running

Table 1 Schematic description of the PF algorithm 
time was proportional to the length $\mathrm{T}$ of the time series, the number of particles $\mathrm{N}$, the number of repetitions $N_{r}$, and it increased quadratically with the number of network nodes [31]. The algorithm was implemented in MATLAB (Mathworks, Natick, MA, U.S.A.) R2017b.

\subsection{Synthetic data}

To validate the proposed approach, two different synthetic networks were used.

- One network with $R=6$ nodes, each with $T=100$ time points, stationary coefficients generated with the MATLAB function varm() with a Signal-to-Noise Ratio (SNR) set to either $\infty\left(\sigma_{\eta}=0\right.$, ideal case $)$ or $6 \mathrm{~dB}$.

- Another network with $R=2$ and $T=250$ was used to assess the PF capability to capture time-varying hidden parameters. In this case, $a_{i j}$ coefficients were zero except for coefficient $a_{21}$, whose value switched from 1 to -1 with a period of 125 time points. The SNR was $10 \mathrm{~dB}$.

The first synthetic dataset allowed us to verify the reliability of the results of the PF and to decide the optimal values of the parameters; the second one allowed us to address the capability of the PF to track variations through time of the AR coefficients.

\subsection{Real fMRI data}

The proposed approach was also retrospectively applied to real fMRI data acquired on healthy volunteers in two different experimental set-ups, with two and four participants respectively, acquired with two-dimensional single-shot echo-planar imaging (EPI) on a 7T MRI system (MR950, GE Healthcare, Chicago, IL, U.S.A.).

- Motor task Time-series consisting of 240 time points with a temporal resolution of $2 s$ were acquired on two subjects with the following acquisition parameters: Time of Echo $(\mathrm{TE})=23 \mathrm{~ms}$, Flip Angle $(\mathrm{FA})=60^{\circ}$, Field of View $(\mathrm{FoV})=(192 \mathrm{~mm})^{2}$, acquisition matrix size $=128 \times 128,32$ slices of thickness $=1.5 \mathrm{~mm}$, resulting in isotropic spatial resolution of $(1.5 \mathrm{~mm})^{3}$ and Time of Repetition (TR) $=2 \mathrm{~s}$. During acquisition, the subjects' thumb- and index-fingertips were stimulated via a pneumatic device (Linari Engineering, Pisa, Italy). The subjects' task was to move the finger whenever it was stimulated. The fMRI data were motion-corrected using MCFLIRT [32]. Spatial smoothing was applied by using a Gaussian kernel of FWHM 3.0mm; each 4D dataset was demeaned and normalized by a single multiplicative factor; high- pass temporal filtering was applied to remove slow temporal drifts of the fMRI signal. Four ROIs were studied covering primary somatosensory (S1), primary motor (M1), supplementary motor (SM) and parietal (P) cortices. All ROIs consisted in four voxels and were manually drawn on each subject on one slice only, to avoid potential slice timing confounds (Fig. 1). The resultant time-series of each ROI were obtained by averaging the four time-series of individual voxels.

- Visual task Time-series with a temporal resolution of 0.8 s were acquired on four trials of either 300 time points (two subjects) or 600 (two subjects). Scanning parameters were $\mathrm{TE}=21 \mathrm{~ms}$, $\mathrm{FA}=48^{\circ}, \quad \mathrm{FoV}=(192 \mathrm{~mm})^{2}$, acquisition matrix size $=64 \times 64,22$ slices of thickness $=3 \mathrm{~mm}$, resulting in isotropic spatial resolution of $(3 \mathrm{~mm})^{3}$ and $\mathrm{TR}=800 \mathrm{~ms}$. The same preprocessing steps described above for the motor task, including motion correction, spatial smoothing, normalization and signal drift removal, were adopted. Subjects underwent a periodic visual stimulation alternating between black and white dots moving along spiral trajectories over a gray background (stimulation $\mathrm{ON}$ ) and presentation of the gray background alone (stimulation OFF). Four ROIs were studied covering the Lateral Geniculate Nucleus (LGN), the Middle temporal cortex (MT), the Primary Visual area (V1) and one con-

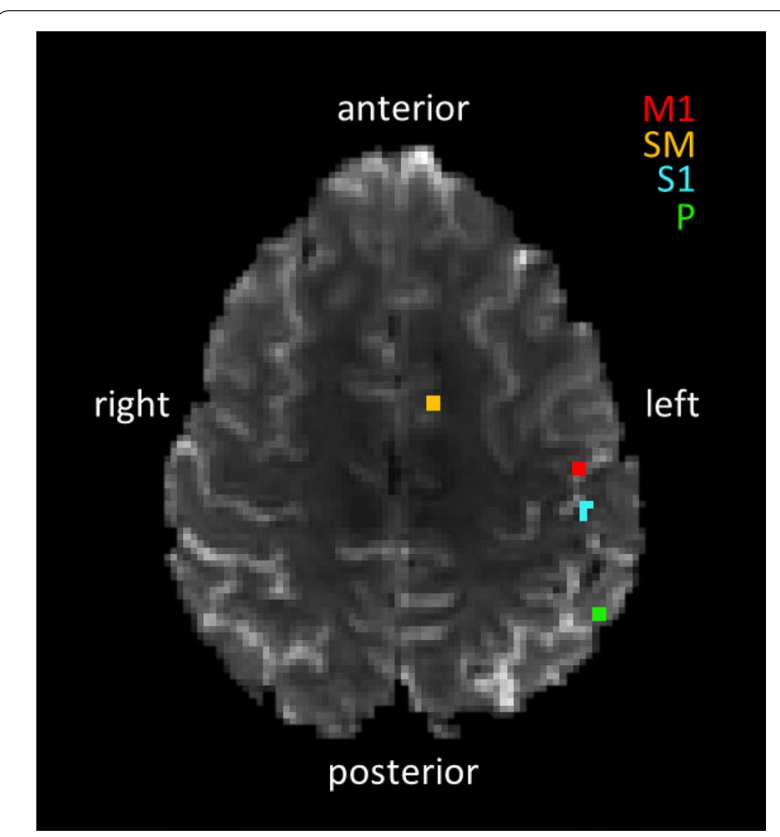

Fig. 1 ROls drawn on one representative subject, representing primary somatosensory (S1), primary motor (M1), supplementary motor (SM) and parietal (P) cortices 
trol ROI in the tempo-parietal cortex (CTRL). Four voxels wide ROIs were manually drawn on each subject on one slice only.

The optimal order of the autoregressive model describing the time series was 1, as estimated by the Schwartz criterion $[12,25,27]$.
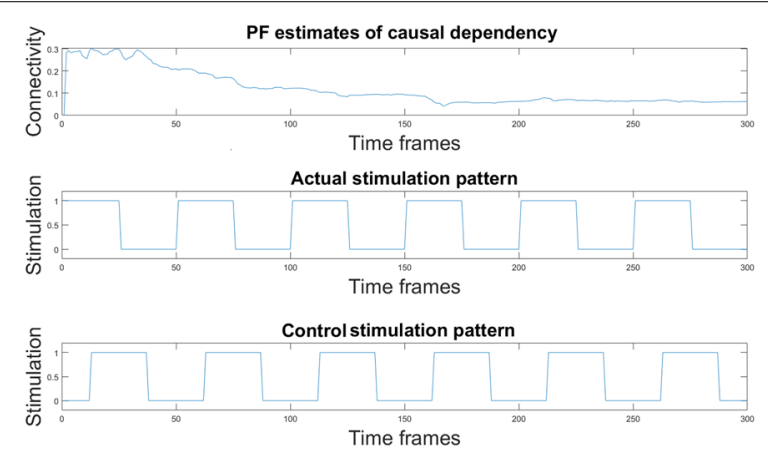

Fig. 2 The plot on top shows the causal dependency of area MT from area $\mathrm{V} 1$ for one of the 4 subjects in the visual task as an example of PF estimates of conditional dependency coefficients between time-series. Plot in the center shows the actual stimulation pattern as a square wave, where the presence and the absence of stimulation are represented by 1 and 0 respectively. The bottom plot shows a time shifted stimulation pattern, which has a half-period offset from the actual stimulation pattern. The t-test was run comparing connectivity values in correspondence of ones (stimulation ON) and zeros (stimulation OFF) in both cases. The second test allowed to exclude spurious changes in connectivity values not due to the stimulation
The particle filter was applied not only to the original time series but also to the same fMRI data shuffled in time. This test allowed to address the effective dependency of the results from the temporal order of the data, i.e. to address causal dependency through time in the data. Subsequently, the $a_{i j}$ coefficients estimated by particle filtering were compared to the delayed correlation (DC) $c_{i j}$ between signals $x_{i}(t)$ and $x_{j}(t-1)$ which reflect the time-invariant conditional correlation between network node (ROI) $j$ over node $i$. Furthermore, the results of the PF were also compared to coefficients estimated in a stationary framework fitting a multivariate linear regression model with stationary coefficients to the data.

With a $t$ test between coefficient values in the presence and in the absence of stimulation we searched statistically significant $(p$-value $<0.05)$ changes in connectivity following the underlying stimulation pattern. Also, as a control analysis, a second t-test was performed, simulating a different stimulation pattern from the actual one, which allowed to exclude variations deriving from spurious fluctuations of the results, as explained in Fig. 2.

\section{Experimental results and discussions}

\subsection{Synthetic data}

Scatter plots in Fig. 3 demonstrate that the conditional dependency coefficients estimated by PF in a stationary network satisfactorily correlate with the true coefficients, both in the noiseless synthetic dataset (Pearson's $\rho=0.96)$ and in the noisy scenario with $\mathrm{SNR}=6 \mathrm{~dB}$ (Pearsons' $\rho=0.59$ ).
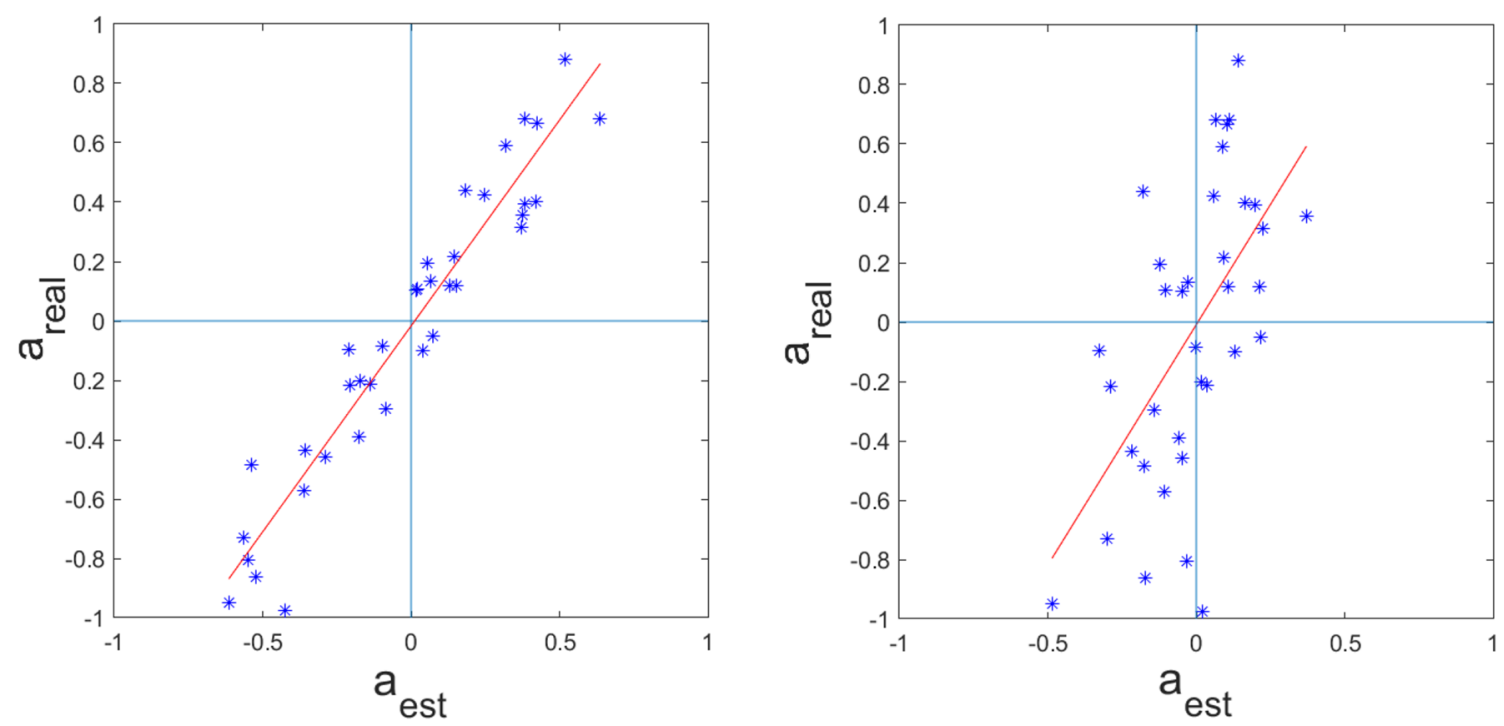

Fig. 3 Scatter plots that relate PF estimates ( $x$ axis) and true values (y axis) of the autoregressive model for a 6-node network with 100 time samples, in the absence of noise (left) and with SNR $=6 \mathrm{~dB}$ (right). The lines are the results of a linear fit of the data: in the noiseless case the slope $m$ and the offset $q$ were 1.39 and $-1.62 \cdot 10^{-2}$, respectively; in the noisy case, $m=1.62$ and $q=8 \cdot 10^{-3}$ 


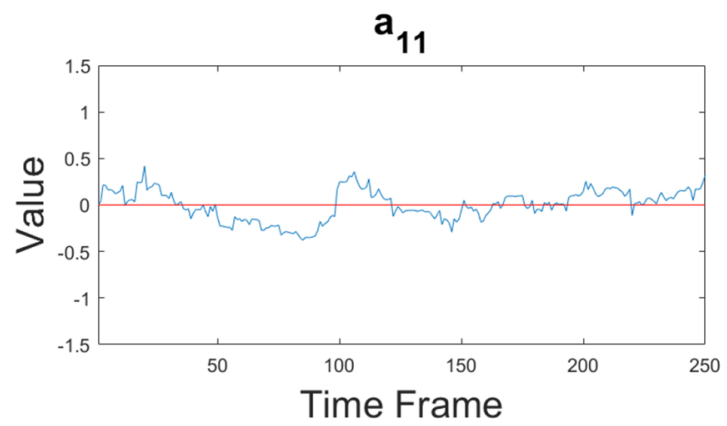

$a_{12}$

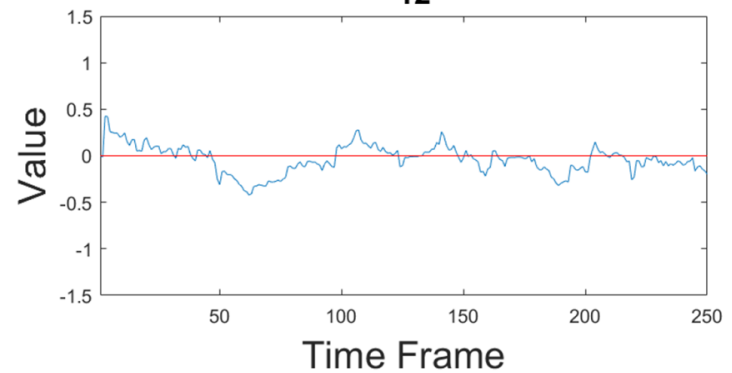

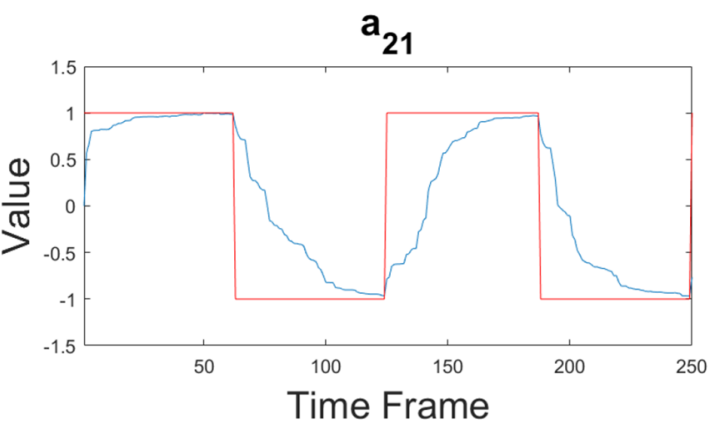

$a_{22}$

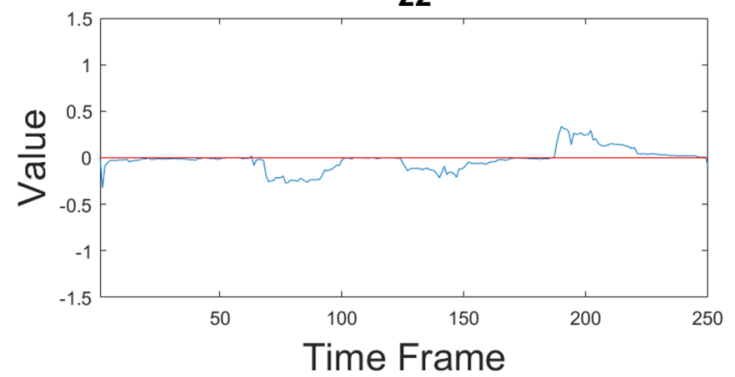

Fig. 4 Time courses of the hidden parameters $a_{i j}$ in the case of a 2-node network with non-stationary coefficient $a_{21}$ alternating between 1 and - 1. Red lines represent the true values, while blue lines represent the estimates obtained by PF

The case of a network with one time-varying coefficient is shown in Fig. 4. The PF tracks the changes of the nonstationary coefficient $a_{21}$, although the estimated values do not immediately follow the abrupt changes between 1 and -1 and viceversa. All the other coefficients are correctly estimated to be close to the nominal null value.

\subsection{Real fMRI data}

\subsubsection{Motor network}

Red lines in Fig. 5 represent average values of the $a_{i j}$ coefficients obtained on fMRI time series and the blue histogram represents the corresponding distribution of mean values of causal interactions for the permuted time series. Since temporal permutation suppresses the causal dependency between subsequent values, it was expected to observe zero-mean Gaussian distributed results, that is, no causal dependency. On the other hand, coefficients representing the causal relationship between two interacting brain areas should have values lying far away from the null distribution. This is what Fig. 5 shows, demonstrating that the particle filter effectively discriminates between unrelated and causally related time series.

The PF captured causal interactions between brain areas, which significantly correlated with a proxy measure of effective connectivity, that is, delayed correlation (DC) $(p<0.001$, Pearson's correlation coefficient $\rho=0.74$, Fig. 6). In particular, in both subjects, the highest $a_{i j}$ coefficients in both PF and delayed correlation were those which represent the conditional dependency of areas M1 and S1, in agreement with current knowledge of brain functioning during a sensory-motor task.

Figure 7 exemplifies the temporal evolution of brain connectivity through three representative $a_{i j}$ coefficients in Subject 2. The top panel displays one representative coefficient involving the control ROI P, which is approximately 0 . Our finding that the S1-on-P coefficient time series exhibits only small fluctuations around zero is in agreement with ground truth that the parietal node is not involved in the motor task. The two bottom panels demonstrate the expected reciprocal influence between M1 and SM areas. It is worth noting that the influences in the two directions, i.e. M1 over SM and vice versa, have different values, as a consequence of the adopted model that allows non-symmetric matrix of coefficients.

\subsubsection{Visual network}

The PF detected causal dependencies between brain areas of the visual network which also correlated with the delayed correlation (Pearson's $\rho=0.70$ and $p<0.001$, as shown in Fig. 8a). Interestingly, all four fMRI datasets showed statistically significant dynamic changes $(p<0.05)$ with a pattern following the underlying stimulation in the effective connectivity coefficient regarding the influence of MT on V1, which are known to take part in the processing of visual stimuli. As an example, Fig. 9 


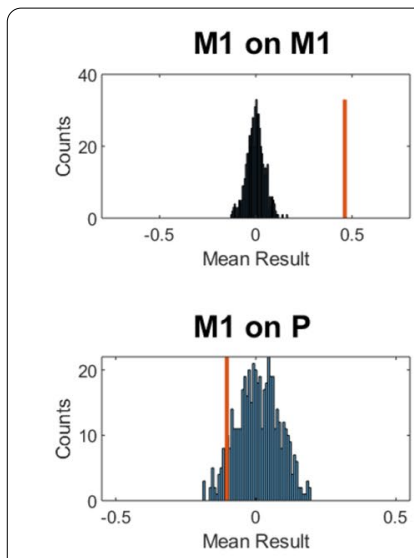

M1 on SM

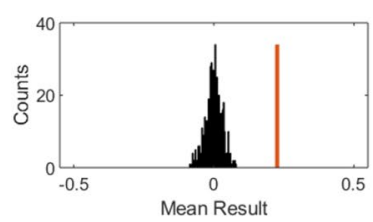

M1 on S1

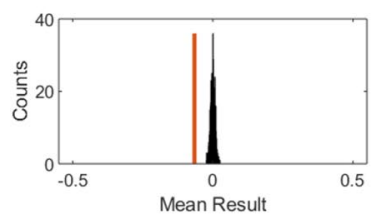

P on M1

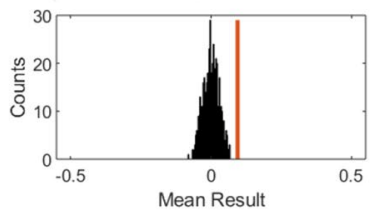

SM on $P$

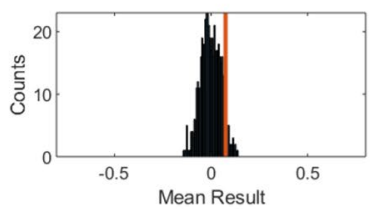

P on SM

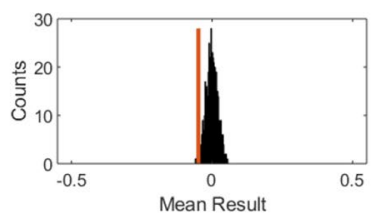

P on S1

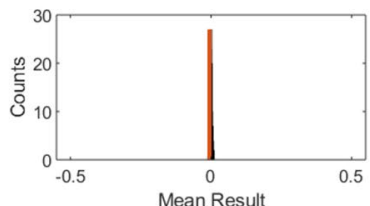

SM on M1

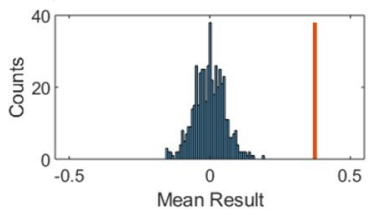

$\mathbf{P}$ on $\mathbf{P}$

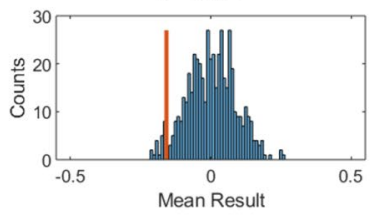

SM on SM

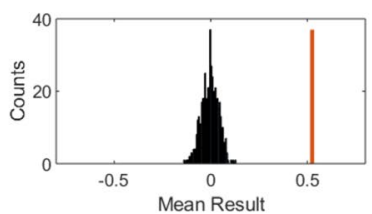

SM on S1

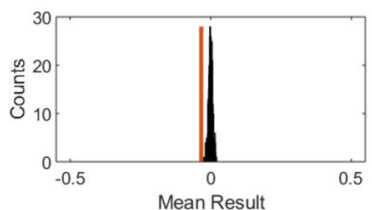

S1 on M1

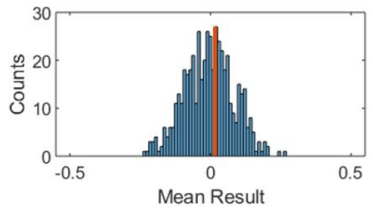

S1 on $P$

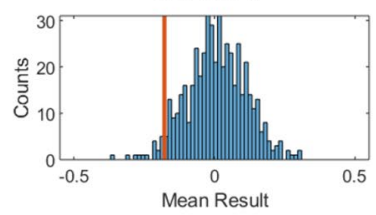

SM on S1

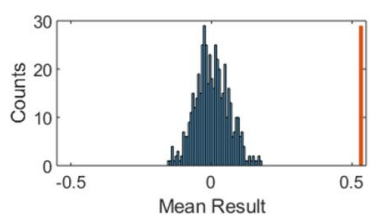

S1 on S1

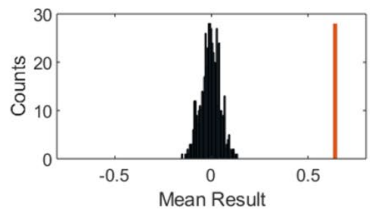

Fig. 5 Mean conditional dependencies between real fMRI data computed through the particle filter: in blue, the histogram of mean values obtained on time-series randomly permuted in time, while the red line shows the corresponding mean value obtained from non-permuted time-series. Many of these values lie well outside of the null distribution, therefore their value reflects the effective mean causal interaction and it is not produced by chance

represents the statistically significant differences in the causal influence of MT on V1 in the presence or absence of the visual stimulus, observed consistently in all four fMRI datasets. Blue crosses show the quality parameter $Q=T / \sigma$, where $T$ is the temporal length of the time series and $\sigma$ is the standard deviation of the data, taken as a measure of noise. $Q$ values indicate a possible explanation for inter-dataset variability: the particle filter performance are enhanced by the timeseries length $(\mathrm{T})$ and reduced by noise (estimated as $1 / \sigma$ ). Not all connectivity coefficients share this same behavior, therefore other factors might influence the results. Figure 10 represents one example of absence of detected causal relationship between area MT and the control region CTRL, and one example of non-symmetrical causality exerted by area $\mathrm{V} 1$ over MT and viceversa.

Figure 8b shows the comparison between results of the PF and stationary AR coefficients (Pearson's $\rho=0.94$ and $p<0.001$ ). A good agreement was found between the two estimates. In addition, changes through time of the
AR coefficient were searched through a sliding-window approach: a multivariate linear regression model was fitted to consecutive and overlapping blocks of 20 time points, and variations in the regression coefficients in phase with the visual stimulation were searched with the same strategy used for the estimates of the PF (Fig. 2). While in this way some coefficients were found to vary, those that did were not the same across the four datasets. Instead, the conditional dependency coefficients estimated with the PF representing the influence of area MT on area V1 were consistently varying in all subjects, suggesting that the PF more reliably detects non-stationarity.

Figure 11 shows the relationship between coefficients obtained by PF and DC with explicit reference to the causal influence they are referred to. We searched for the appropriate clustering of the data in Fig. 11. The number of clusters was chosen as the knee of the number-ofclusters vs distance-to-every-centroid curve. The optimal number was 3 and each cluster's centroid was plotted as a black cross in Fig. 11. All coefficients representing the 


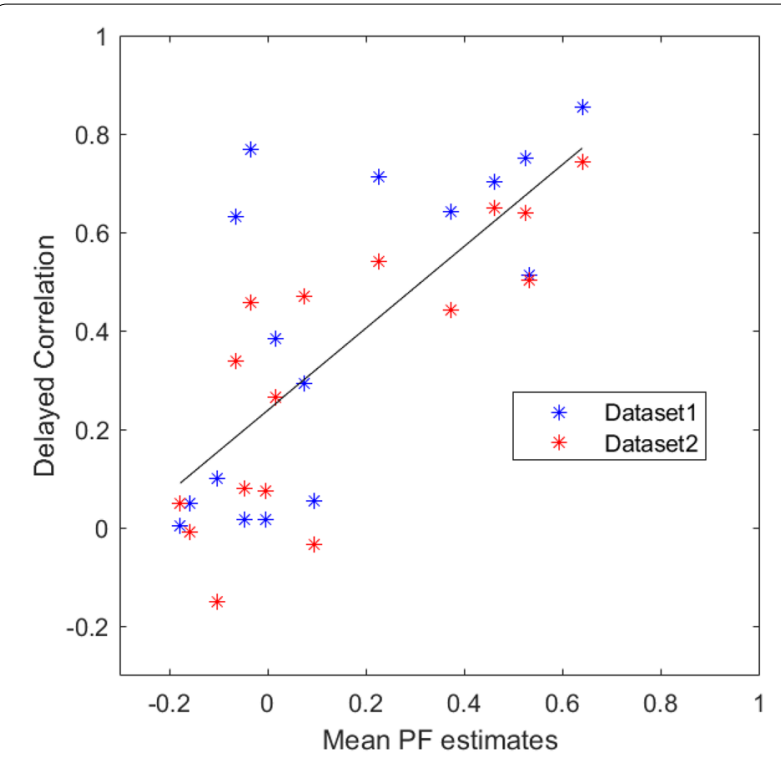

Fig. 6 Scatter plot showing the relationship between mean PF estimates (horizontal axis) and delayed correlation (vertical axis) on the two sensory-motor experiments. On both results taken altogether the Pearson's correlation coefficient $\rho$ is 0.74 , which corresponds to a statistically significant correlation with $p<0.001$. Slope and offset of the linear fit were 0.83 and 0.24 respectively

causal interactions involving the CTRL region (black symbols) belong to cluster $\mathrm{A}$, that is, small values of DC and mean PF estimates, except for the self-causality terms, which all lay in the vicinity of the $C$ centroid. Every non-diagonal coefficient involving ROIs LGN, MT and V1 belong to the cluster B. In particular, in Fig. 11, the horizontal red line and vertical green line intersect in the point equally distant to the centroids $A$ and $B$. All values representing the causal influence of MT on V1 (which vary following the stimulation pattern, represented by highlighted red diamonds in the figure) and that of V1 on MT (green stars) lay right to the green line and above the red line.

\section{Discussion}

Other implementations of SMC algorithms were previously proposed to investigate brain connectivity in fMRI data. Murray and Storkey [33] proposed a forwardbackward Particle Filter using, as observation equation, a stochastic extension of the balloon model, which was proposed to describe the haemodynamics that follow brain activity [34]. In their study, the hidden parameters of the model resulted approximately constant, probably as a consequence to the complexity of the model itself $[35,36]$.

In a different study, Ahmad et al. [37] adopted a symmetric, linear, first-order, time-varying Autoregressive (TVAR) model and used a Rao-Blackwellized PF to estimate the temporal relationships among fMRI time-series representing four brain regions during resting state. The assumption of symmetry in coupling coefficients, that is $a_{i j}=a_{j i}$, reduced model complexity, but did not permit

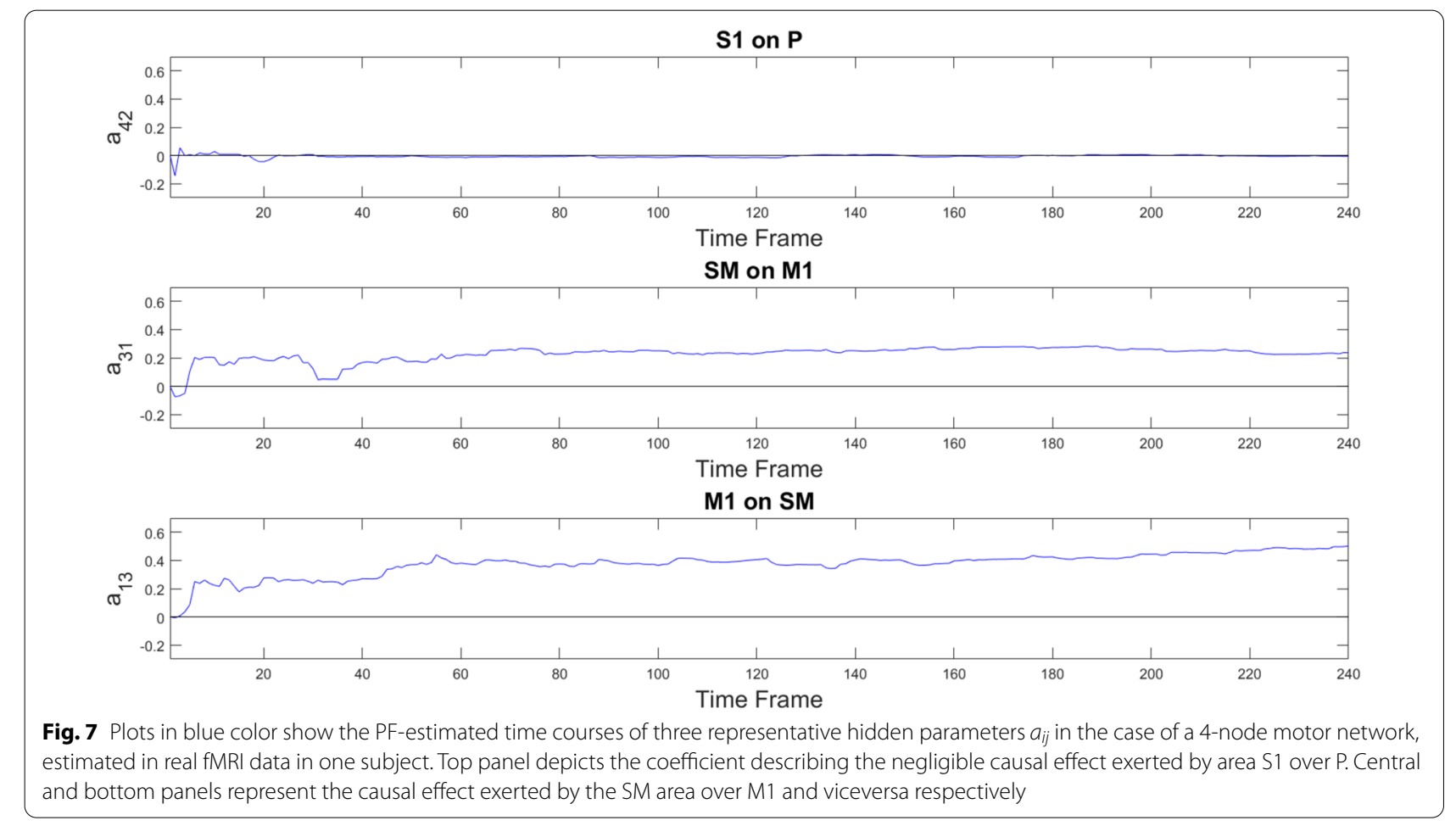




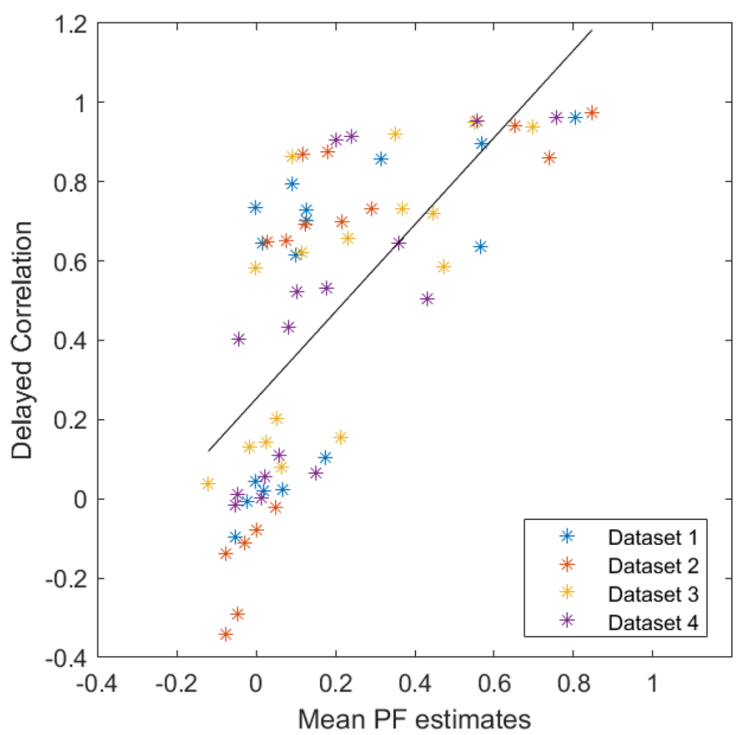

(a)

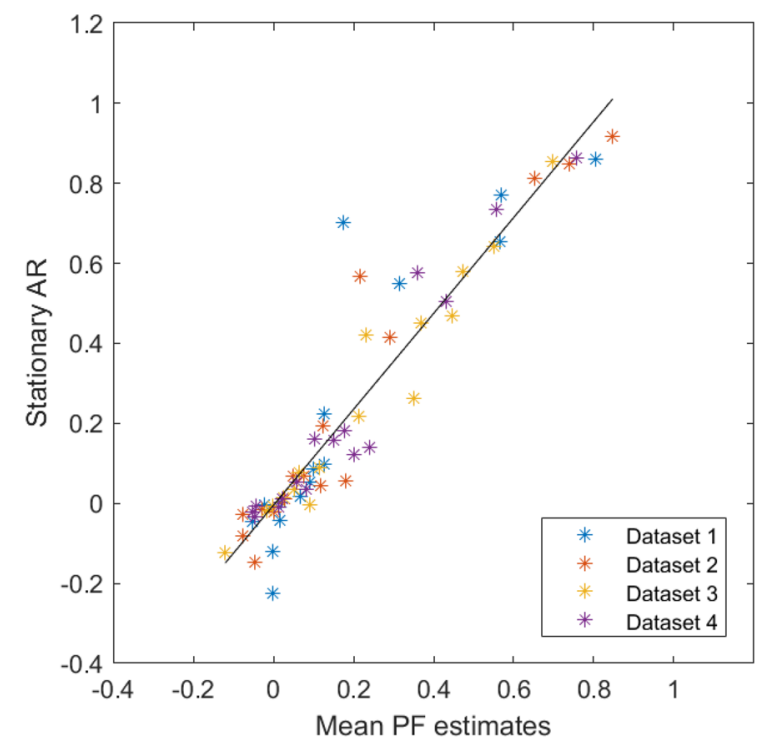

(b)

Fig. 8 a Scatter plot between mean PF estimates (horizontal axis) and delayed correlation (vertical axis) on the four visual experiments fMRI data. A $p<0.001$ was obtained with a Pearson's correlation coefficient $\rho=0.70$. The black line is the result of a linear fit. Slope and offset of the linear fit were 1.10 and 0.25 respectively. b Scatter plot between average PF estimates (horizontal axis) and stationary AR coefficients (vertical axis). The resulting Pearson's correlation coefficient $\rho$ was 0.94 , with a $p<0.001$. The black line is the result of a linear fit, with slope and offset 1.2 and -0.004 respectively

to infer neither the directionality of the network nor any possibly asymmetric cause/effect interaction between brain areas. Therefore their approach cannot be used to investigate effective connectivity. Also, the results were

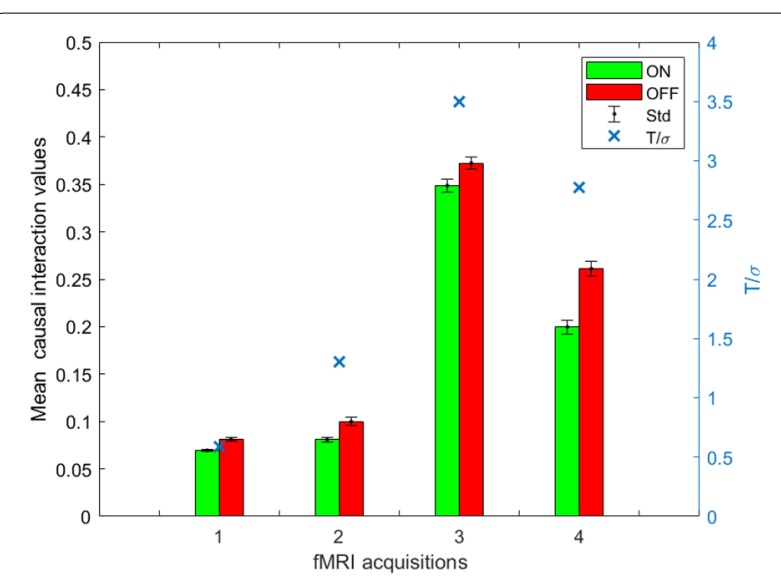

Fig. 9 Comparison between presence (green bar) and absence (red bar) of visual stimulation for the mean $a_{i j}$ coefficient representing the causal influence of MT on V1 in the four different datasets. Bars indicate the mean values and the standard deviation of the mean. Higher values of the coefficients are obtained in data sets with better quality parameter $Q$, as shown by blue crosses

not benchmarked with the outcome results from different analyses and the resting-state paradigm did not allow any analysis on the temporal evolution of the results.

In our implementation on fMRI data, the time-averaged PF estimates were in agreement with a proxy measure of causality, that is, delayed correlation. Part of the mismatch between the proposed method and delayed correlation could be explained by the fact that the PF algorithm studies the network as a whole and produces estimates of $a_{i j}$ coefficients that update at every time instant, while delayed correlation is a measure of pairwise causality that does not take into account possible non-stationarities and spurious cause-effect relationships mediated by other nodes of the network.

In the first experimental setup involving the motor network, statistically significant changes in connectivity were not found, but the poor temporal resolution of the data $(2 s)$ may have prevented the detection of these changes.

On the contrary, in our study of the visual network statistically significant changes in connectivity were found in four different datasets with a pattern following the underlying stimulation, without requiring any previous knowledge on the actual stimulation paradigm during the estimation process. These variations interested the influence of MT on V1 which is consistent with our understanding of cortical processing in the early visual cortex [24]. The average behavior of PF's results and a simpler regression estimate were in good agreement, but the PF also enabled to report consistent results across different datasets in the same experimental paradigm. Furthermore, while the PF is "blind", sliding window analysis 


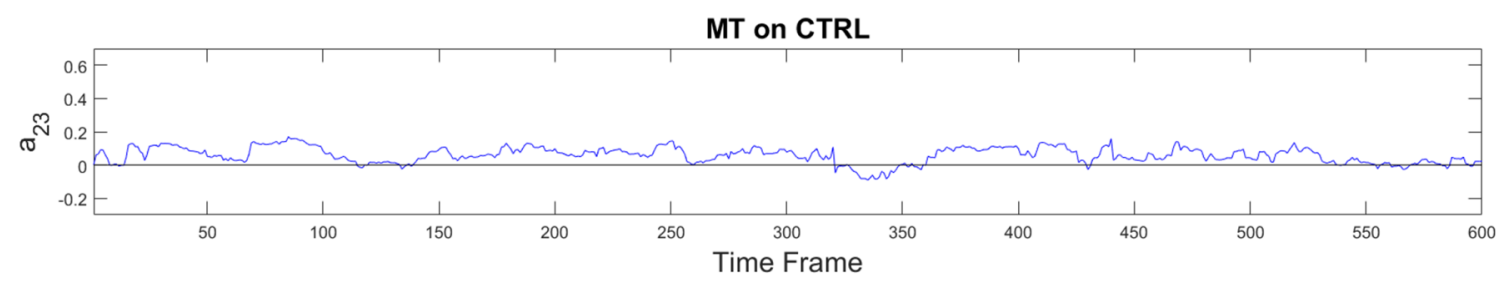

V1 on MT

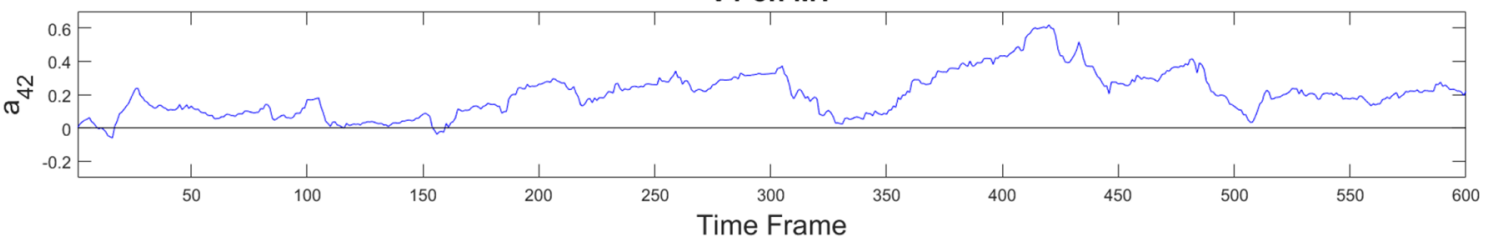

MT on V1

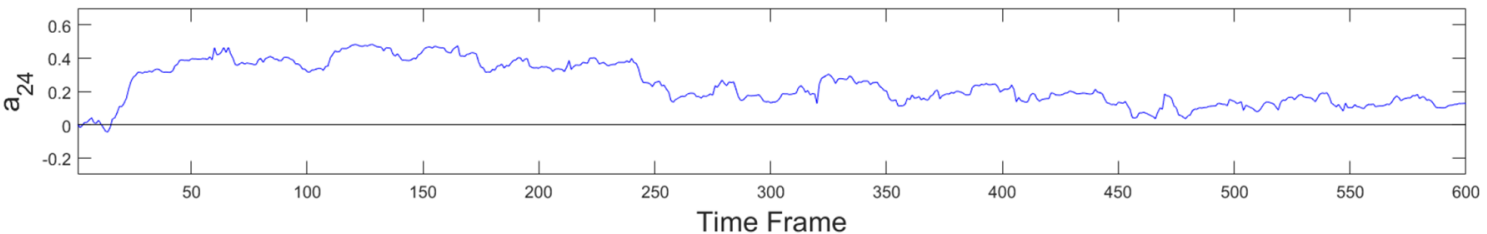

Fig. 10 Plots in blue color show the PF-estimated time courses of three representative hidden parameters $a_{i j}$ in the case of the 4-node visual network, estimated in real fMRI data in one subject. Top panel depicts the coefficient describing the negligible causal effect exerted by area MT over the control area. Central and bottom panels represent the non-symmetrical causal effect exerted by the V1 area over MT and viceversa respectively

requires the inclusion of additional pieces of information, such as the stimulation paradigm timing, which in some cases may not be available.

The stability of the PF-estimated coefficients at larger time scales is in agreement with the regime of stationarity, commonly adopted in functional and effective connectivity fMRI studies: indeed, our experiments demonstrate a good agreement between time-averaged values of $a_{i j}$ coefficients and both delayed correlation and stationary AR coefficients. This finding suggests that on large temporal scales the brain network has stable interactions within its nodes, under the assumption of linear first order autoregression.

Mean conditional dependency coefficients were found to vary between different datasets. As Fig. 9 shows, in some cases these differences can be explained through the joint contribution of noise and temporal length of the data, i.e. the quality parameter $Q=T / \sigma$. The brain haemodynamic responses may also be involved, which vary not only among subjects but also between different areas in the same subject [38], which was not taken into account in this study.

\section{Conclusions}

We used Particle Filter to test and identify the time-varying brain connectivity as evidenced in fMRI images. Our experiments confirmed the hypothesis of time-varying brain connectivity pattern and gave evidence for nonsymmetric connectivity. It was possible to detect statistically significant changes in cortical cause-effect relationships correlated with the underlying task-rest pattern during the fMRI acquisition.

Future studies should test the performance of the proposed algorithm in fMRI experiments with higher time resolution, namely $<0.8 s$, and they should aim to unveil possibly asymmetric changes in effective connectivity among brain regions. Also, to minimize the impact of vascular dynamics and highlight neural ones, future studies should use more sophisticated experimental designs that enable a better control over the non-uniformity of brain haemodynamics across different areas [38-40].

As suggested by Bugallo and Djuric [41], the PF can be improved by a parallel implementation when dealing with complex system, such as the brain. Ordinary brain connectivity analysis represents ROIs as time series obtained by averaging signals originating from more voxels of that region. This helps improving the Signal-to-Noise Ratio (SNR) but assumes some wide-scale connectivity features. Because of this wide-scale connectivity assumption, more reliable results may be achieved with a parallel combination of Particle Filters carried over single-voxels time series.

Our results, together with the possibility to refine the methodology, suggest that the proposed computational 


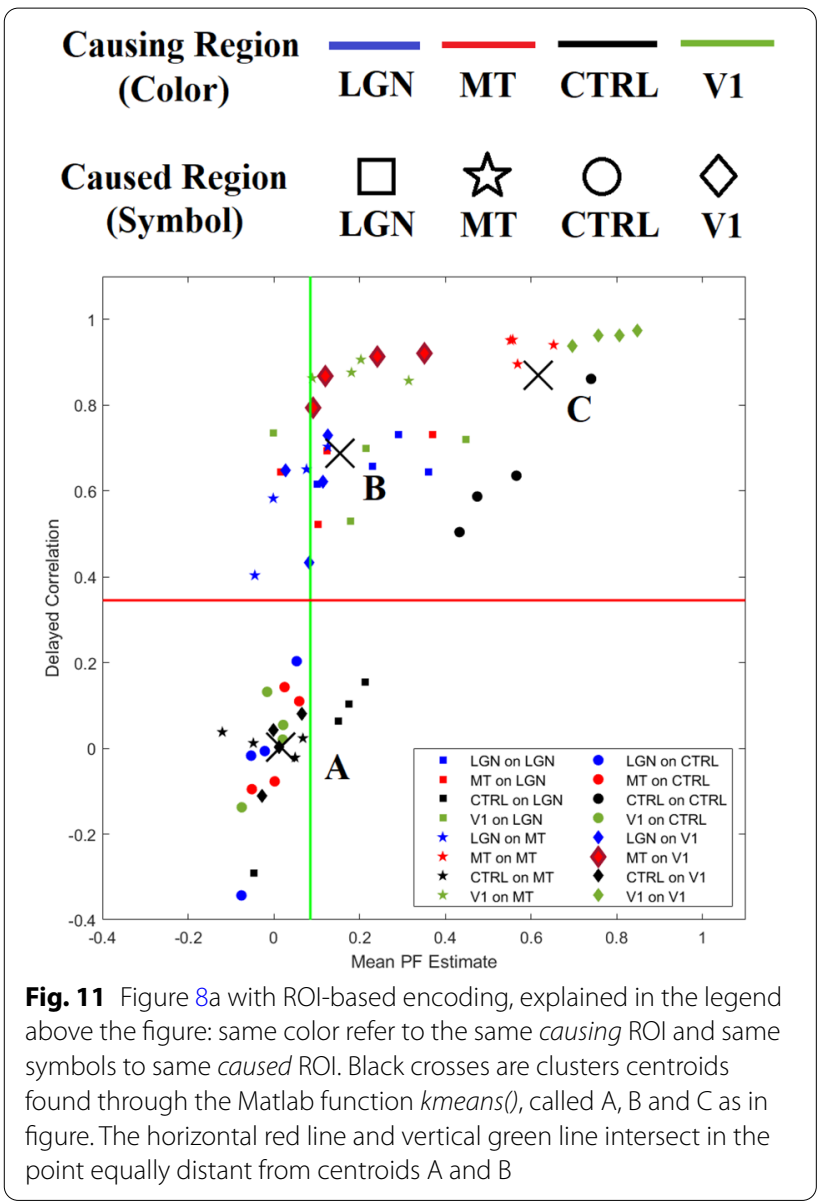

method, the Particle Filter, can be capable to infer timevarying effective connectivity on acquisitions with acceptable scan duration, without the need of any constraint or previous knowledge about the examined network or timing of the underlying brain processes.

\section{Acknowledgements}

The authors thank Dr Sergiy Ancherbak for having shared with them his particle filtering code for time-dependent gene network modeling.

\section{Authors' contributions}

Conceived and designed the experiments: PA, MC, MT. Performed the experiments: MC, LB, GB. Analyzed the data: PA, MC. Contributed analysis tools: EK. Wrote the paper: PA, MC, EK. All authors read and approved the final manuscript

\section{Funding}

This work has been partially supported by grants "RC 2018-2020" and " 5 per mille" to IRCCS Fondazione Stella Maris, funded by the Italian Ministry of Health.

\section{Availability of data and materials}

The fMRI data used in this study will be made available upon direct request to the authors.

\section{Declarations}

Ethics approval and consent to participate

Ethics approval was issued by "Comitato per la sperimentazione clinica dei medicinali dell'Azienda Ospedaliero Universitaria Pisana", reference number 12697, and by "Comitato Etico Regionale per la Sperimentazione Clinica della Regione Toscana", reference number 32891. All subjects provided written informed consent

\section{Competing interests}

The authors declare that they have no competing interests.

\section{Author details \\ 'Department of Neuroscience, Psychology, Pharmacology and Child Health, University of Florence, Florence, Italy. ${ }^{2}$ Department of Neurosciences, Rehabili- tation, Ophthalmology, Genetics, and Maternal-and-Child Sciences, University of Genoa, Genova, Italy. ${ }^{3}$ Laboratory of Medical Physics and Magnetic Reso- nance, IRCCS Stella Maris, Pisa, Italy. ${ }^{4}$ Tsinghua-Berkeley Shenzhen Institute, Data Science and Information Technology Center, Shenzhen, China. ${ }^{5}$ Imago 7 Research Center, Pisa, Italy. Information Science and Technology Institute (ISTI), National Council of Research (CNR), Pisa, Italy.}

Received: 29 January 2021 Accepted: 16 August 2021

Published online: 29 September 2021

\section{References}

1. Friston KJ (2011) Functional and effective connectivity: a review. Brain Connect 1:13-36

2. Stephan KE, Friston KJ (2010) Analyzing effective connectivity with functional magnetic resonance imaging. WIREs Cognit Sci 1:446-459

3. Greicius MD, Srivastava G, Reiss AL, Menon V (2004) Default mode network activity distinguishes Alzheimer's disease from healthy aging: evidence from functional MRI. Proc Natl Acad Sci USA 101:4637-4642

4. Greicius MD (2008) Resting-state functional connectivity in neuropsychiatric disorders. Curr Opin Neurol 21:424-430

5. Jones DT, Vemuri P, Murphy MC, Gunter JL, Senjem ML, Machulda MM et al (2012) Non-stationarity in the "resting brain's"' modular architecture." PLOS ONE 7:e39731

6. van den Heuvel MP (2010) Exploring the brain network: a review on resting-state fMRI functional connectivity. Eur Neuropsychopharmacol 20:519-534

7. Greicius MD, Flores BH, Menon V, Glover GH, Solvason HB, Kenna H et al (2007) Resting-state functional connectivity in major depression: abnormally increased contributions from subgenual cingulate cortex and thalamus. Biol Psychiatry 62:429-437

8. Jirsa V, Mclntosh AR (2007) Models of effective connectivity in neural systems. In: Jirsa V, Mclntosh AR (eds) Handbook of brain connectivity, 5th edn. Springer, Berlin, pp 303-326

9. Honey G, Bullmore E (2004) Human pharmacological MRI. Trends Pharmacol Sci 25(7):366-374

10. Stephan KE, Harrison LM, Penny WD, Friston KJ (2004) Biophysical models of fMRI responses. Curr Opin Neurobiol 14:629-635

11. Gottesman II, Gould TD (2003) The endophenotype concept in psychiatry: etymology and strategic intentions. Am J Psychiatry 160:636-645

12. Roebroeck A, Formisano E, Goebel R (2005) Mapping directed influence over the brain using Granger causality and fMRI. Neuroimage 25:230-242

13. Desphande G, Sathian K, Hu X (2010) Effect of hemodynamic variability on Granger causality analysis of fMRI. Neuroimage 52:884-896

14. Leonardi N, Van De Ville D (2015) On spurious and real fluctuations of dynamic functional connectivity during rest. Neurolmage 104:430-436

15. Friston KJ, Harrison L, Penny W (2003) Dynamic causal modelling. Neuroimage 19:1273-1302

16. Geyer CJ (2011) Introduction to Markov chain Monte Carlo. In: Brooks S, Gelman A, Jones G, Meng X (eds) Handbook of Markov chain Monte Carlo. CRC Press, Taylor and Francis Group, Boca Raton 
17. Ancherbak S, Kuruoğlu EE, Vingron M (2016) Time-dependent gene network modeling by Sequential Monte Carlo. IEEE/ACM Trans Comput Biol Bioinform 13:1183-1193

18. Ambrosi P, Costagli M, Kuruoglu EE, Biagi L, Buonincontri G, Tosetti M (2019) Investigating time-varying brain connectivity with functional magnetic resonance imaging using sequential Monte Carlo. In: 2019 27th European Signal Processing Conference (EUSIPCO). A Coruna: IEEE ( $p$. $1-5)$

19. Djuric PM, Kotecha JH, Zhang J, Huang Y, Ghirmai T, Bugallo MF, Miguez J (2003) Particle filtering. IEEE Signal Process Mag 20:19-38

20. Arulampalam MS, Maskell S, Gordon N, Clapp T (2002) A tutorial on particle filters for nonlinear/non-gaussian bayesian tracking. IEEE Trans Signal Process 50:174-188

21. Costagli M, Kuruoğlu EE (2007) Image separation using particle filters. Digital Signal Process 17:935-946

22. Wang Z, Kuruolu EE, Yang X, Xu Y, Huang TS (2011) Time varying dynamic Bayesian network for nonstationary events modeling and online inference. IEEE Trans Signal Process 59(4):1553-1568

23. Valdés-Sosa PA, Roebroeck A, Danizeau J, Friston K (2011) Effective connectivity: influence, causality and biophysical modeling. Neuroimage 58:339-361

24. Gaglianese A, Costagli M, Bernardi G, Ricciardi E, Pietrini P (2012) Evidence of a direct influence between the thalamus and hMT+ independent of $V 1$ in the human brain as measured by fMRI. Neuroimage 60:1440-1447

25. Casorso J, Kong X, Chi W, Van De Ville D, Yeo BTT, Liègeois R (2019) Dynamic mode decomposition of resting-state and task fMRI. Neuroimage 194:2-54

26. Bressler SL, Seth AK (2011) Wiener-Granger causality: a well-established methodology. Neuroimage 58(2):323-9

27. Gaglianese A, Costagli M, Ueno K, Ricciardi E, Bernardi G, Pietrini P, Cheng $K$ (2015) The direct, not V1-mediated, functional influence between the thalamus and middle temporal complex in the human brain is modulated by the speed of visual motion. Neuroscience 284:833-844

28. Doucet A, Godsill S, Andrieu C (2000) On sequential Monte Carlo sampling methods for Bayesian filtering. Stat Comput 10:197-208

29. Liu JS, Chen R (1995) Blind deconvolution via sequential imputations. J Am Stat Assoc 90:567-576

30. Gençağa D, Kuruoğlu EE, Ertüzün A (2010) Modeling non-Gaussian timevarying vector autoregressive processes by particle filtering. Multidim Syst Sign Process 21:73
31. Mohammadi A, Asif A (2013) Distributed particle filter implementation with intermittent/irregular consensus convergence. IEEE Trans Signal Process 61(10):2572-2587

32. Jenkinson M, Bannister P, Brady M, Smith S (2002) Improved optimisation for the robust and accurate linear registration and motion correction of brain images. Neurolmage 17(2):825-841

33. Murray L, Storkey AJ (2008) Continuous time particle filtering for fMRI. Adv Neural Inf Process Syst 20:1049-1056

34. Buxton RB, Wong EC, Frank LR (1998) Dynamics of blood flow and oxygenation changes during brain activation: the balloon model. Magn Reson Med 39(6):855-864

35. Hettiarachchi IT, Mohamed S, Nahavandi S (2012) Identification of nonlinear fMRI models using auxiliary particle filter and Kernel smoothing method. In: 34th Annual International Conference of the IEEE EMBS, San Diego, California: IEEE. 28 Aug-1 Sept

36. Chambers M, Wyatt C (2011) An analysis of blood-oxygen-leveldependent signal parameter estimation using particle filters. In: 2011 IEEE International Symposium on Biomedical Imaging. New York: IEEE. Mar 30 (p. 250-253)

37. Ahmad MF, Murphy J, Vatansever D, Stamatakis EA, Godsill S. (2015) Tracking changes in functional connectivity of brain networks from resting-state fMRI using Particle Filters. IEEE International Conference on Acoustics, Speech and Signal Processing. New York: IEEE

38. Handwerker DA, Ollinger JO, D'Esposito M (2004) Variation of BOLD hemodynamic responses across subjects and brain regions and their effects on statistical analyses. Neuroimage 21:1639-1651

39. Duggento A, Passamonti L, Valenza G, Barbieri R, Guerrisi M, Toschi N (2018) Multivariate Granger causality unveils directed parietal to prefrontal cortex connectivity during task-free MRI. Sci Rep 8:1-11

40. Aguirre GK, Zarahn E et al (1997) Empirical analyses of BOLD fMRI statistics: II. Spatially smoothed data collected under null-hypothesis and experimental conditions. Neurolmage 5(3):199-212

41. Bugallo MF, Djuric PM. Complex systems and particle filtering, 42nd Asilomar Conference on Signals, Systems and Computers. New York: IEEE; 2008

\section{Publisher's Note}

Springer Nature remains neutral with regard to jurisdictional claims in published maps and institutional affiliations.

\section{Submit your manuscript to a SpringerOpen ${ }^{\circ}$ journal and benefit from:}

- Convenient online submission

- Rigorous peer review

- Open access: articles freely available online

- High visibility within the field

Retaining the copyright to your article

Submit your next manuscript at springeropen.com 